\title{
A case of bipolar I disorder with suspected impairment of the thermoregulation center induced by neuronal desynchrony within evening oscillator for which aripiprazole was effective
}

\author{
Tetsuo Tashiro* \\ Tashiro Clinic, Akita-heal Medical Corporation, Japan
}

\begin{abstract}
This patient with bipolar I disorder experienced a peculiar sleep/wake circadian rhythm (desynchrony of evening/morning oscillators of suprachiasmatic nucleus (SCN)) during a depressive episode, which was thought to be an SCN coupling disorder (Tashiro, 2017, JSR). Desynchrony was seen between rectal temperature rhythm and sleep rhythm in this patient, and desynchrony was also seen between them and the day-night cycle. In addition, the circadian rhythm of temperature exhibited pronounced lengthening/shortening in a short period of time, the oscillation of which alternated daily between normalization and flattening. These were believed to have been caused by neural desynchrony within SCN neuronal groups (especially evening oscillator). The rectal temperature circadian rhythm showed a phase advance of 297 min with bright light therapy, but the sleep-wake cycle and hypersomnic depressive state did not improve. The patient's sleep cycle disorder and symptoms of depression improved in 2 weeks after resuming aripiprazole $24 \mathrm{mg}$. With respect to the rectal temperature circadian rhythm during a period of recovery 2 years later, oscillation improved, it synchronized with sleep, and it entrained to the day-night cycle. It is surmised that the inhibitory effect of aripiprazole on GSK3 $\beta$ increased expression of the clock gene Bmal1 and enhanced SCN coupling. The patient's hypersomnic depressive state and circadian rhythm disorders resolved with improvement of the SCN coupling impairment (desynchrony between and within SCN neuronal groups). In addition, improvement in the functional connectivity between the thermoregulation center and the sleep-promoting center may have prevented recurrence of manic-depressive episodes for an extended period of time ( 3 years).
\end{abstract}

\section{Introduction}

Biological clock abnormalities are often found during depressive episodes in manic-depressive or depressive patients. Examples of biological clock abnormalities include patients with external desynchrony between the day-night cycle and the biological clock $[1,2]$ and patients with internal desynchrony in whom the core temperature rhythm (autonomic nervous system activity) and timing of hormonesecreting tissue activity and organ activity differ from those of healthy individuals [3].

With respect to the biological clock, separation of the nocturnal sleep bout that each occurred at different circadian rhythms was seen in this patient during a depressive episode, although it only lasted for around 1 week. A phenomenon in which the first half and latter half of the nocturnal active phase occur at different circadian rhythms and gradually split due to impairment of neural coupling in the SCN is known to occur in nocturnal rodents [4-6]. The separation of the nocturnal sleep bout seen in the present case was similar to this phenomenon. The present author previously reported in JSR [2] that what likely occurred in the present case was impairment of SCN neural coupling causing desynchrony between SCN evening/morning oscillators, with the first half and latter half of the sleep bout oscillating at separate rhythms. To the best of my knowledge, this is the first report of this phenomenon in humans; therefore, further analysis of this patient is important.

In this report, I primarily examine a peculiar rectal temperature circadian rhythm (autonomic nervous system) abnormality that occurred during a depressive episode in which the sleep bout was not split, and I discuss impairment of the functional connectivity between the thermoregulation center and the sleep-promoting center, which are located closely together in the preoptic area of the brain, and the importance in terms of the pathology of impairment of the SCN coupling in depression and depressive state.

\section{Case}

The patient is a Japanese male in his 40 s who was diagnosed with bipolar I disorder (DSM-IV). His mother suffers from major depressive disorder. He had been engaged in programming-related work at a major information technology (IT) company. Treatment for affective disorder began in March 2000. He was hospitalized for treatment of manic and depressive episodes. Due to these episodes, he was not able to live on his own. In July 2005, he began visiting a day care center every day and receiving treatment at our clinic. Hypersomnia and lying in bed all day continued for over a year. He was unable to wake up in

Correspondence to: Tetsuo Tashiro, Tashiro Clinic, Akita-heal Medical Corporation 1-23-1 Higashidori, Akita City, Akita,010-0003, Japan, Tel: +8118-884-1500; Fax: +81-18-884-1501; E-mail: akitattashiro@hotmail.co.jp

Key words: bipolar disorder, circadian rhythm, body temperature regulation, suprachiasmatic nucleus, functional connectivity

Received: December 01, 2017; Accepted: December 26, 2017; Published: December 29, 2017 
Tashiro T (2017) A case of bipolar I disorder with suspected impairment of the thermoregulation center induced by neuronal desynchrony within evening oscillator for which aripiprazole was effective

the morning, and his father would drive him to the day care center due to his drowsiness, where he would spend 6 hours in the daytime with 30 persons. The patient's condition is characterized by prolonged mild depressed mood, hypersomnia, diminished ability to think, and hypobulia.

With respect to the sleep circadian rhythm disorders, the sleep journal revealed two normal sleep periods (each lasting about 3 months) and a hypersomniac depressive period lasting 27 months in total during the 33 months prior to administration of aripiprazole. During prolonged depressive episodes with hypersomnia, which lasted for 463 days, various circadian rhythm sleep disorders, such as delayed sleep phase, polyphasic sleep, separation of the sleep bout resembling splitting and circabidian rhythm $(48 \mathrm{~h})$, were seen. No abnormal findings were seen on physical examinations and head MRI.

With respect to pharmacotherapy, using sodium valproate 800 $\mathrm{mg}$ as the base drug, an atypical antipsychotic (olanzapine $20 \mathrm{mg}$ or quetiapine $300 \mathrm{mg}$ ) and an antidepressant (mianserin $30 \mathrm{mg}$, trazodone $75 \mathrm{mg}$, or fluvoxamine $50 \mathrm{mg}$ ) were taken as add-on drugs, but none of them was very effective for the patient's depression or sleep circadian rhythm disorders, suggesting the refractory nature of this case.

The patient had a strong desire to see the treatment succeed and took it upon himself to look for a better treatment, and bright light therapy was subsequently initiated at the request of the patient. The patient measured his rectal temperature himself by inserting a probe into the rectum at home. At the time of temperature measurement initiation, the setting of the measuring instrument and the insertion method (10 centimeters in depth) of a probe to anus were taught to the patients in the hospital. The employed highly precise temperature logger is LT-8 (resolution: 0.01 degrees Celsius, measurement period: 20 seconds) made in Gram corporation.

Informed consent was obtained from the patient in writing for treatment, tests, and this case report. Note that some modifications have been made to the text to protect the anonymity of the patient.

\section{Rectal temperature rhythm}

Rectal temperature was measured at home during the depressive episode and the recovery state. Sleep diagrams for both periods with the days that rectal temperature was measured are shown in Figure 1. During the hypersomnic depressive episode, the sleep/wake rhythm was very irregular. The patient was taking sodium valproate $1000 \mathrm{mg}$, vitamin B12 $100 \mathrm{mg}$ and quetiapine $600 \mathrm{mg}$, which had been fixed 5 months previously. Rectal temperature was measured for 5 days beginning on Day 17 after adding ramelteon (melatonin agonist) $8 \mathrm{mg}$, and bright light therapy (10000 Lux in front of eyes immediately after waking, $30 \mathrm{~min}$ ) was subsequently added, but the sleep/wake rhythm had not improved by Day 48. During the 3-year recovery state while taking aripiprazole, the sleep/wake rhythm was synchronized with the day-night cycle and stable. The patient was taking aripiprazole $24 \mathrm{mg}$ and sodium valproate $1000 \mathrm{mg}$.

Data that were thought to be poor measurements were excluded from the analysis of body temperature data. Data during times the temperature probe was removed to bathe and defecate, and data during times of elevated body temperature due to contact with warm water (approx. 1-hour period after bathing), were excluded.

In Figure 2, during the depressive episode, body temperature increased beginning immediately after sleep onset on Days 1-3, the opposite of what is seen in normal individuals. A decrease in body temperature appeared in the latter half of sleep, and the trough (minimum) was seen when getting up or after getting up (Days 2-4). Desynchrony was seen between body temperature and sleep rhythm, as well as with the day-night cycle. Oscillation of the body temperature circadian rhythm alternated every other day from a day where it was about $1.5^{\circ} \mathrm{C}$ to a day where a decrease in body temperature associated with sleep onset was missing and the temperature curve was almost flat.

Body temperature trough-related data are shown in Table 1. The trough occurred 77 min and 32 min later on Days 2 and 3, respectively, as compared with the day before, and a very pronounced advance (297 $\mathrm{min}$ ) was seen on Day 5. Exposure to bright light immediately after waking from nocturnal sleep may have triggered this sudden pronounced advance in the body temperature phase. Nevertheless, neither the sleep/wake circadian rhythm disorder nor the symptoms of depression improved despite the rapid advance in the body temperature phase (Figure 1).

During the 3-year recovery state while taking aripiprazole, the sleep/wake circadian rhythm was synchronized with the day-night cycle and stable. In terms of rectal temperature, as shown in Figure 2, normal synchronization with sleep was seen, as well as synchronization with the day-night cycle.

\section{Discussion}

There was a report of various body temperature circadian rhythm abnormalities in patients with depression/depressive state [7]. In addition, it was reported that remission of depression was maintained for about 2 weeks by advancing sleep and synchronizing it with temperature rhythm [8], and that remission of depression was achieved by advancing the body temperature and melatonin secretion circadian rhythms with morning light therapy and synchronizing them with the sleep/wake rhythm in seasonal depression with delayed circadian rhythm of the biological clock [9]. Thus, it has been theorized that body temperature circadian rhythm abnormalities are associated with the etiology of depression.

The SCN, which is the center of circadian rhythm expression, switches between activity/rest in the evening and morning, and is set so that autonomic nervous system activity, hormone secretion, and visceral activity are activated at the optimal phase for expression of physical function. There are thought to be evening/morning oscillators in the SCN, but their location is unknown even in rodents. However, Inagaki et al. [10] identified two sites in the SCN of mice where the peak time of clock gene Per1 expression differed, and they surmised that these might be evening/morning oscillators. It is known that desynchrony of evening/morning oscillators occurs in AVP-Bmal1-/mice with SCN coupling impairment [6]. The author previously speculated that impairment of SCN coupling resulted in desynchrony of so-called evening/morning oscillators (the night sleep bout split in two parts, with each expressing a different circadian rhythm) in the SCN neuronal groups during a depressive episode in this patient [2].

Table 1. Trough of rectal temperature circadian rhythm and its relation to sleep bout.

\begin{tabular}{|c|c|c|c|c|c|}
\hline \multirow{2}{*}{ Days } & \multirow{2}{*}{ Light therapy } & \multicolumn{2}{|c|}{ Trough } & \multirow{2}{*}{ Phase shift of trough } & \multirow{2}{*}{ Relation to sleep } \\
\hline & & time & ${ }^{\circ} \mathbf{C}$ & & \\
\hline $1^{\text {st }}$ & & $8: 40$ & 35.77 & & late half of sleep \\
\hline $2^{\text {nd }}$ & & $9: 57$ & 36.40 & delay (77 min) & after sleep \\
\hline $3^{\text {rd }}$ & 0 & $10: 29$ & 35.93 & delay (32 min) & end of sleep \\
\hline $4^{\text {th }}$ & 0 & 9:09 & 36.61 & advance $(-80 \mathrm{~min})$ & end of sleep \\
\hline $5^{\text {th }}$ & & $4: 11$ & 35.60 & advance $(-298 \mathrm{~min})$ & early half of sleep \\
\hline
\end{tabular}

Trough of rectal temperature circadian rhythm and its relation to nocturnal sleep bout in a depressive phase 
Tashiro T (2017) A case of bipolar I disorder with suspected impairment of the thermoregulation center induced by neuronal desynchrony within evening oscillator for which aripiprazole was effective

\section{sleep diagram}

\section{a) depressive hypersomnic phase (ramelteon $8 \mathrm{mg}$ administration)}

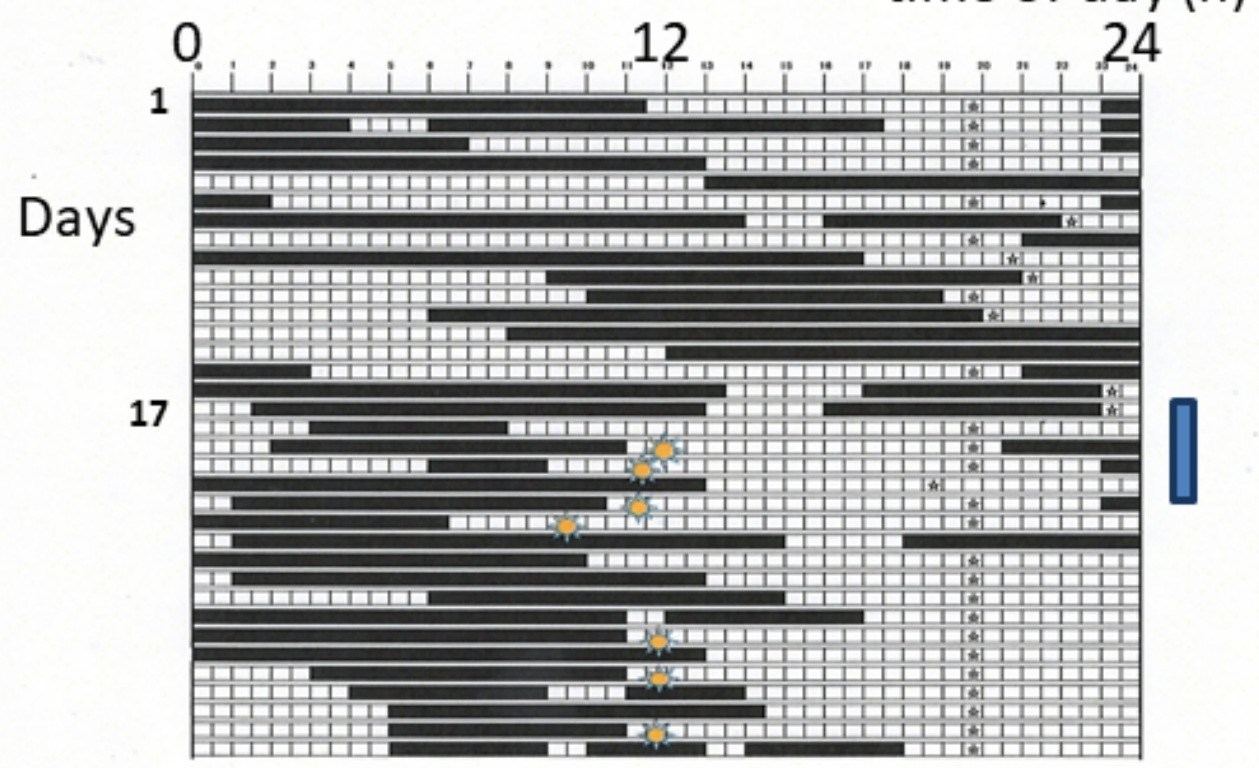

b) recovery state (APZ 24mg administration)

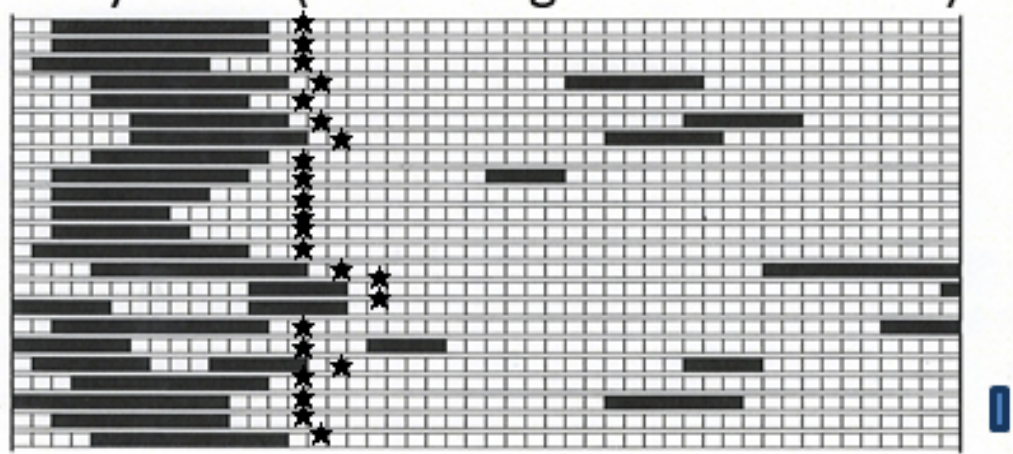

\section{ing : bright light exposure (30min $10000 \mathrm{Lx})$ 々 : ramelteon dosing}

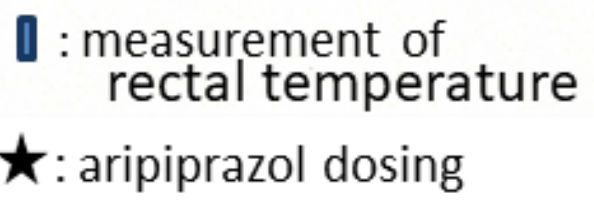

Figure 1. Sleep diagrams for the mild hypersomnic depressive episode and the recovery state are shown together with days rectal temperature was measured. During the hypersomnic depressive episode, the sleep rhythm was very irregular with desynchrony between it and the day-night cycle. Rectal temperature was measured for 5 days beginning on Day 17 after adding ramelteon $8 \mathrm{mg}$. The patient received 10,000 lux of light therapy for $30 \mathrm{~min}$ immediately after getting up beginning on Day 19 . The sleep/wake circadian rhythm did not improve with these treatments. During the recovery state while taking aripiprazole $24 \mathrm{mg}$, the sleep/wake circadian rhythm showed stable nocturnal sleep despite occasional naps lasting 2-3 hours, as well as re-entrainment with the day-night cycle.

It is known that the temperature rhythm exhibits a relatively stable circadian rhythm due to SCN regulation. In the present case, days where the temperature curve was almost flat occurred every other day during depressive episodes when the sleep bout was not split. Refinetti et al. [11] reported that suprachiasmatic lesions eliminated circadian rhythmicity of body temperature in golden hamsters. In these hamsters, high body temperature seen in the activity phase was stable and continued for 24 hours beginning immediately after the procedure, and the phenomenon of body temperature decreasing in the sleep phase had disappeared. The flattening of the temperature curve seen in the present case was similar to this phenomenon in hamsters with suprachiasmatic lesions. Thus, the weak output from the evening oscillator that occurred because of desynchrony within the evening oscillator neuronal group in the present case might not have properly 


\section{Depressive hypersomnic phase (ramelteon 8mg administration)}

recovery state

(APZ 24 mg administration)

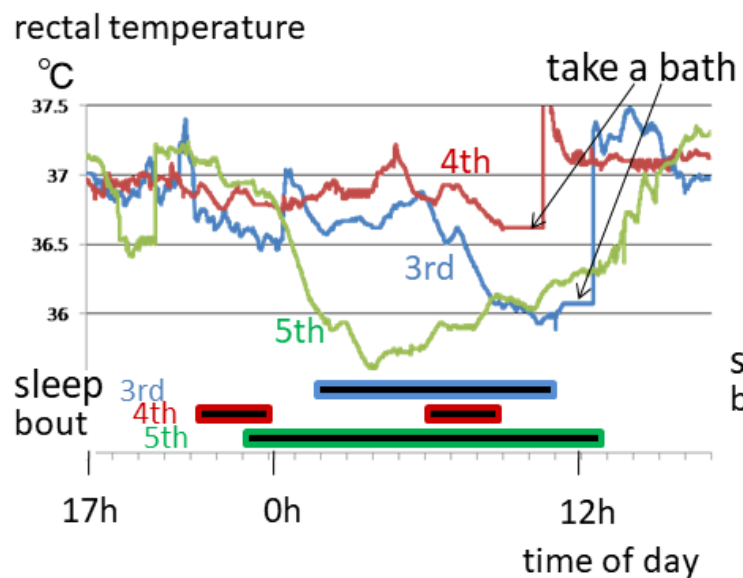

rectal temperature

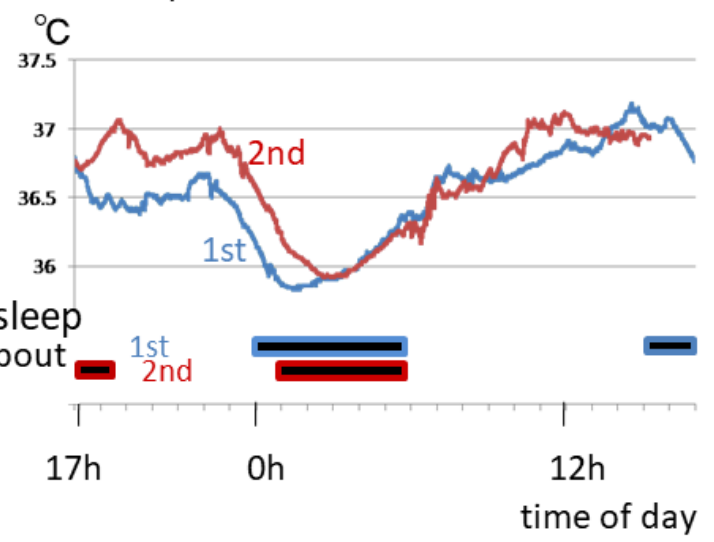

Figure 2. Temperature and sleep/wake circadian rhythms during hypersomnic depressive phase and recovery state Body temperature circadian rhythm (upper row) and sleep bout (lower row) during the hypersomnic depressive episode (left) and recovery state (right) are shown. The horizontal axis indicates a 24-hour period. The probe was removed to bathe and defecate, and the missing data were supplemented with body temperature data taken at the time the probe was removed. For the depressive phase, data for measurement Days 3-5 are shown. The probe moved to the vicinity of the anus due to large bowel peristalsis sometime between 6:00 p.m. and 8:00 p.m. on the 5th day, and it was inserted to the proper depth at 8:00 p.m. Therefore, measurements recorded during this time period were regarded as poor measurements. During the depressive phase, desynchrony was seen between body temperature and sleep rhythm, as well as with the day-night cycle. Oscillation of the body temperature circadian rhythm alternated every other day from a day where it was about $1.5^{\circ} \mathrm{C}$ to a day where the temperature curve was almost flat. During the recovery state, as to body temperature, normal synchronization with sleep was seen, as well as entrainment with the day-night cycle.

initiated the thermoregulation center at times, resulting in absence of a decrease in body temperature and flattening of the body temperature curve. An unstable temperature rhythm caused by phase advances and delays of short duration and an excessive phase shift reaction to bright light were also seen during this time. These abnormalities in this case may have been due to an SCN coupling disorder. Yamada et al. [12] reported that the degree of the body temperature phase shift reaction was greater in patients with non-seasonal affective disorder than in healthy subjects as a result of morning/evening bright light therapy. Thus, impairment of SCN coupling may be viewed as an underlying mechanism in depressive episodes.

Desynchrony within evening/morning oscillator neuronal groups may cause phase anomalies at various brain sites/bodily systems that switch day and night. Specifically, the functional connectivity between the sleep-promoting center and the thermoregulation center, which are thought to be located in the preoptic area of the brain, was impaired in the present case, and impairment of the SCN as the central clock may also be involved in the functional connectivity abnormalities between many brain regions seen in depression/depressive state [13].

APZ has a partial agonistic effect on 5-HT1A/2C/7 receptors [14], and upregulates release of histamine in the anterior hypothalamus [15]. Therefore, photo-like/non-photoic phase advance of the circadian rhythm can occur with administration of aripiprazole [2,16-18]. This phase advance effect might improve hypersomnic depression, but the hypersomnic depression did not improve despite bright light therapy having a pronounced phase advance effect in the present case. It is known that impairment of SCN coupling occurs in mice when there is a lack of Bmallin SCN neurons [6]. As reported previously, a phenomenon similar to the phenomenon in which evening/morning oscillators showed a different oscillation rhythm in AVP-Bmal1-/mice occurred in this case with withdrawal of APZ. Furthermore, this phenomenon rapidly improved with resumption of APZ [2]. In addition to the above, APZ may improve impairment of SCN coupling
[2] because it increases the expression of clock gene Bmal1 [19] by having an inhibitory effect on GSK3 $\beta$ in the molecular clock of SCN [20]. Based on these findings, it is conjectured that enhancement of SCN coupling via the inhibitory effect of aripiprazole on GSK3 $\beta$ played an important role in improving the various circadian rhythm disorders and improving and preventing the depression/depressive state in the present case.

More specifically, the peculiar sleep circadian rhythm disorder (desynchrony of evening/morning oscillators) and hypersomnic depressive symptoms in this patient improved almost simultaneously about 2 weeks after resuming aripiprazole [2]. Therefore, improving impairment of SCN coupling (desynchrony between and within SCN neuronal groups) and normalizing its function as a central clock with administration of aripiprazole resulted in a stable circadian rhythm (external/internal resynchronization) characterized by functional recovery of the thermoregulation center and the sleep-promoting center, normalization and synchronization of the functional connectivity between the two, and also maintenance of daytime wakefulness and entrainment with the day-night cycle. These findings are consistent with the fact that aripiprazole improved the patient's depression and prevented recurrence of manic-depressive episodes for an extended period of time (3 years).

\section{Limitation}

The discussion of the pathological mechanism was limited to presentation of data from only one case. This pathological mechanism will need to be further confirmed in the future as more animal experiments and clinical studies are conducted.

\section{Acknowledgements}

I express my deep gratitude to Dr Yasuo Hishikawa, Professor Emeritus of Akita University, for proofreading this paper, and to Dr 
Tashiro T (2017) A case of bipolar I disorder with suspected impairment of the thermoregulation center induced by neuronal desynchrony within evening oscillator for which aripiprazole was effective

Tetsuo Shimizu, Professor of Akita University, for providing valuable advice.

\section{Conflict of interest}

I have not received any financial support nor had any relationships that may pose a conflict of interest. I have not received financial support from any company whose product figures prominently in this submitted manuscript nor from any company making competing products.

\section{References}

1. Kripke DF, Mullaney DJ, Atkinson M, Wolf S (1978) Circadian rhythm disorders in manic-depressives. Biol Psychiatry 13: 335-351. [Crossref]

2. Tashiro T (2017) Improvement of a patient's circadian rhythm sleep disorders by aripiprazole was associated with stabilization of his bipolar illness. J Sleep Res 26: 247-250. [Crossref]

3. Wehr TA, Goodwin FK (1981) Biological rhythms and psychiatry, American Handbook of Psychiatry. ( $\left.2^{\text {nd }} E d n\right)$ Basic Books, New York pp: 46-74.

4. Pittendrigh CS, Daan S (1975) A functional analysis of circadian pacemakers in nocturnal rodents. J comp Physiol 106: 333-355.

5. Golombek DA, Rosenstein RE (2010) Physiology of circadian entrainment. Physiol Rev 90: 1063-1102. [Crossref]

6. Mieda M, Ono D, Hasegawa E, et al. (2015) Cellular clocks in AVP neurons of the SCN are critical for interneuronal coupling regulating circadian behavior rhythm. Neuron 85 : 1103-1116. [Crossref]

7. Goodwin FK, Jamison KR (2007) Manic-Depressive Illness: Bipolar Disorders and Recurrent Depression. (2nd Edn) Oxford University Press, New York, pp: 667-672.

8. Wehr TA, Wirz-Justice A, Goodwin FK, Duncan W, Gillin JC (1979) Phase advance of the circadian sleep-wake cycle as an antidepressant. Science 206: 710-713. [Crossref]

9. Lewy AJ, Sack RL, Singer CM, White DM (1987) The phase shift hypothesis for bright light's therapeutic mechanism of action: theoretical considerations and experimental evidence. Psychopharmacol Bull 23: 349-353. [Crossref]
10. Inagaki N, Honma S, Ono D, Tanahashi Y, Honma K (2007) Separate oscillating cell groups in mouse suprachiasmatic nucleus couple photoperiodically to the onset and end of daily activity. Proc Natl Acad Sci U S A 104: 7664-7669. [Crossref]

11. Refinetti R, Kaufman C M, Menaker M (1994) Complete suprachiasmatic lesions eliminate circadian rhythmicity of body temperature and locomotor activity in golden hamsters. J Comp Physiol A 175: 223-232. [Crossref]

12. Yamada N, Martin-Iverson MT, Daimon K, Tsujimoto T, Takahasi S (1995) Clinica and chronobiological effects of light therapy on nonseasonal affective disorders Biological Psychiatry 37:866-873.

13. Zeng L, Shen H, Liu L, Wang L, Li B, et al. (2012) Identifying major depression using whole-brain functional connectivity: a multivariate pattern analysis. Brain 135: 14981507. [Crossref]

14. Shapiro DA, Renock S, Arrington E, Chiodo LA, Liu L, et al. (2003) Aripiprazole, a novel atypical antipsychotic drug with a unique and robust pharmacology. Neuropsychopharmacology 28: 1400-1411. [Crossref]

15. Murotani T, Ishizuka T, Isogawa Y, Karashima M, Yamatodani A (2011) Possible involvement of serotonin 5-HT2 receptor in the regulation of feeding behavior through the histaminergic system. Neuropharmacology 61: 228-233. [Crossref]

16. Cote NK, Harrington ME (1993) Histamine phase shifts the circadian clock in a manner similar to light. Brain Res 613: 149-151. [Crossref]

17. Sprouse J, Li X, Stock J, McNeish J, Reynolds L (2005) Circadian rhythm phenotype of 5-HT7 receptor knockout mice: 5-HT and 8-OH-DPAT-induced phase advances of SCN neuronal firing. J Biol Rhythms 20: 122-131. [Crossref]

18. Cuesta M, Clesse D, Pévet P, Challet E (2009) New light on the serotonergic paradox in the rat circadian system. J Neurochem 110: 231-243. [Crossref]

19. Sahar S, Zocchi L, Kinoshita C, Borrelli E, Sassone-Corsi P (2010) Regulation of BMAL1 Protein Stability and Circadian Function by GSK3b-Mediated Phosphorylation. PLoS ONE 5: e8561. [Crossref]

20. Park SW, Lee JG, Ha EK, Choi SM, Cho HY, et al. (2009) Differential effects of aripiprazole and haloperidol on BDNF-mediated signal changes in SH-SY5Y cells. Eur Neuropsychopharmacol 19: 356-362. [Crossref]

Copyright: (C2017 Tashiro T. This is an open-access article distributed under the terms of the Creative Commons Attribution License, which permits unrestricted use, distribution, and reproduction in any medium, provided the original author and source are credited. 